\title{
A remark on R. G. Woods' paper "The minimum uniform compactification of a metric space"
}

(Fund. Math. 147 (1995), 39-59)

by

\section{G. Charalambous (Samos)}

\begin{abstract}
A question raised in R. G. Woods' paper has a simple solution.
\end{abstract}
The minimum uniform compactification $u X$ of a metric space $X$ is the smallest compactification of $X$ such that every bounded real-valued uniformly continuous function on $X$ has a continuous extension to $u X$. Two subsets of $X$ are distant iff they have disjoint closures in $u X$. Woods proves that $u \mathbb{R}$ is a perfect compactification of $\mathbb{R}$ and leaves the case of $u \mathbb{R}^{n}$ open.

TheOREm. Let $X$ be a convex subset of a normed linear space. Then $u X$ is a perfect compactification of $X$.

Proof. Let $f: \beta X \rightarrow u X$ be the Stone-Cech extension of the inclusion $X \rightarrow u X$. In what follows the bar ${ }^{-}$will denote closure in $u X . u X$ is a perfect compactification of $X$ iff $f$ has connected fibers. Suppose that $u X$ is not perfect. Then there is a point $p$ of $u X-X$ such that the closed subspace $f^{-1}(p)$ of $\beta X$ is not connected. Consequently, $f^{-1}(p)$ is the union of non-empty disjoint closed subsets $E, F$ of $\beta X$. As $\beta X$ is normal, there are disjoint open subsets $G, H$ of $\beta X$ such that $E \subset G$ and $F \subset H$. Let $A=X-G \cup H$. Now the image under $f$ of the compact space $\beta X-G \cup H$ is a closed subset of the Hausdorff space $u X$ containing $A$ but not $p$. Hence $p \notin \bar{A}$. Let $B$ be an open neighbourhood of $p$ in the regular space $u X$ such that $\bar{A} \cap \bar{B}=\emptyset$. Then $B \cap X=B_{1} \cup B_{2}$, where $B_{1}=B \cap X \cap G$ and $B_{2}=B \cap X \cap H$.

As $B$ is open and $X$ is dense in $u X$, we have $\bar{B}=\overline{B \cap X}=\bar{B}_{1} \cup \bar{B}_{2}$. Thus, without loss of generality, we may assume that $p \in \bar{B}_{1}$. Note that $p$ also belongs to $f(F)$ and hence to the bigger set $\overline{H \cap X}$. Consequently, $d\left(B_{1}, H \cap X\right)=0$, where $d$ is the metric induced by a norm $|\cdot|$ on $X$.

1991 Mathematics Subject Classification: 54D35, 54E35. 
Let $\varepsilon>0$. Then there are $b$ in $B_{1}$ and $c$ in $H \cap X$ such that $d(b, c)<\varepsilon$. Consider next the line segment $L=\{(1-t) b+t c: 0 \leq t \leq 1\}$ joining $b$ to $c$ in the convex set $X$. As $L$ is a connected subspace of $\beta X$ and $G, H$ are disjoint open sets of it containing $b, c$, respectively, $A=X-G \cup H$ contains at least one point $a=(1-t) b+t c$ of $L$. But then $d(a, b)=|a-b|=$ $|-t b+t c|=t d(b, c)<\varepsilon$. This implies $d(A, B)=0$ and hence $\bar{A} \cap \bar{B} \neq \emptyset$.

This contradiction establishes the result.

Department of Mathematics

University of the Aegean

Karlovassi 83200 Samos, Greece

Received 18 October 1995;

in revised form 12 December 1995 